\title{
Homenaje de la Facultad de Arquitectura, Diseño y Estudios Urbanos de la pUc al arquitecto Horacio Borgheresi
}

\author{
Fernando Pérez Oyarzun ${ }^{1}$
}

La posibilidad de dar un testimonio sobre el arquitecto Horacio Borgheresi con ocasión de este homenaje, es también una invitación a que otros hagan lo mismo. Tal posibilidad nos hace volver la vista hacia la facultad de la memoria, sin duda un componente fundamental de nuestra humanidad. Descuidar nuestra memoria es descuidar esa misma humanidad; olvidar a otros es olvidarnos a nosotros mismos ya que gracias a ellos somos lo que somos. Por ello, mientras nos sea concedida la gracia de la memoria es nuestro deber ejercerla y defender nuestra historia de la amenaza del olvido. Decir cómo se ha visto a alguien cercano y cuánto uno le debe no sólo lo honra al homenajeado: nos honra a nosotros mismos.

Horacio Borgheresi era decano de la Facultad de Arquitectura de la Pontificia Universidad Católica de Chile cuando ingresé a la escuela en 1968. Había asumido ese cargo después de que Sergio Larraín lo ejerciera por 15 años, encabezando un recordado proceso de modernización de la enseñanza. No era poca responsabilidad. Era el año siguiente de la reforma de 1967. Quienes recién ingresábamos estrenábamos algunas de las novedades académicas que la reforma había traído consigo: un nuevo currículo, nuevos profesores. La Escuela de Arquitectura de la puc había estado en los inicios del movimiento. Tal circunstancia había conducido a Fernando Castillo Velasco, miembro de un comité de reforma, a la rectoría. Por todas partes se respiraba un aire de cambio no exento de tensiones.

Un decano es una figura impresionante y muchas veces lejana para un estudiante recién ingresado. Sin embargo, mi condición de delegado de los estudiantes de primer año me obligó a tratar con él con frecuencia, en reuniones personales o en consejos o asambleas académicas. Lo recuerdo como un personaje afable, intuitivo, de reacciones rápidas no siempre meditadas y elegante.

La crisis que se produjo en la facultad en 1969 multiplicó para mí las oportunidades de encuentro. Fui uno de los tres estudiantes que formaban parte de un comité que analizaba las oportunidades que se abrían para la escuela luego de una grave crisis interna que terminaría poniendo fin al decanato de Borgheresi. Recuerdo múltiples reuniones: en la escuela, en el estadio Santa Rosa, en el café Villareal en la calle Pedro de Valdivia o en su propia casa. Esta última ha permanecido para mí como una de las impresiones arquitectónicas que marcaron mi formación. Ubicada en lo que entonces era la calle Lo Saldes ${ }^{2}$ se entraba a ella por un pasaje adoquinado cubierto de vegetación hasta alcanzar el acceso. La casa, un volumen simple, se ubicaba al fondo del terreno y lo dividía en dos: al norte, un pequeño patio empedrado asoleado y repleto de macetas; al sur, una superficie de césped sombreada y rodeada de arbustos. La sala de estar daba a ambos patios y tenía por tanto doble orientación. "No concibo una sala de estar sin doble orientación" me dijo en una ocasión Horacio en una de sus típicas y definitivas sentencias, las mismas que me han dado vueltas en la cabeza por años y sin duda han influido al decidir algún proyecto. La sala tenía un cielo bajo de madera muy wrightiano, con un lucernario un poco más elevado al centro; en un rincón se encontraba la zona del comedor donde recuerdo una enorme fotografía de John Wayne que hablaba de las filiaciones norteamericanas de la sensibilidad de Horacio. No he podido olvidar esa casa.

Al dividirse la escuela en tres departamentos independientes y paralelos, opté por el Departamento de Arquitectura de Obras para continuar mis estudios. La opción por un grupo de profesores frente a otro no fue fácil para mí ni para los restantes estudiantes. Es probable que mi relación con Horacio y mi confianza en sus puntos de vista hayan influido en ella. El asumió como director del departamento y por tanto continuó siendo mi autoridad académica. A mi vez, yo continué siendo delegado estudiantil debiendo por tanto asistir a múltiples consejos y reuniones. Así transcurrieron los años que van de 1970 a 1973. No fueron tiempos fáciles: la actividad académica estaba permanentemente atravesada por tensiones internas y externas. En las múltiples discusiones que con ocasión de ello se generaron no siempre estuvimos de acuerdo; lo recuerdo porque no es bueno borrar esas diferencias y esas distancias, especialmente en la universidad. A pesar de ello, jamás dejé de apreciarlo y admirarlo como arquitecto y como profesor.

El segundo semestre de 1973 tomé con él mi último taller en la escuela. Las circunstancias no eran fáciles: yo había sido parte de una serie de talleres experimentales que Juan Baixas había dirigido a su vuelta de Francia, los que no siempre habían sido bien comprendidos dentro del Departamento de Arquitectura de Obras. Cuando escogí ese taller lo hice con el propósito declarado de demostrar que el interés que habíamos puesto en dichos talleres en los aspectos constructivos de la arquitectura, no necesariamente contradecía nuestro interés ni capacidad para el diseño. Recuerdo ese último taller como uno de los que me dio más satisfacciones en toda mi formación. Sin duda la presencia permanente y no pocas veces cariñosa de Horacio jugó un rol central en esa experiencia. La casa de ladrillo que proyecté entonces, literalmente inspirada en las casas patios de Mies y no sin una cierta referencia a la casa de Horacio en Lo Saldes, permanece como uno de los buenos recuerdos de mi paso por la escuela. Ella fue juzgada en una comisión de examen en la que participaban Sergio Rojo y Enrique Browne. Todavía conservo entre mis papeles un dibujo suyo a mano alzada con las proporciones y detalles de una chimenea que delineó en una de las correcciones de tablero y poco después utilicé en lo que fue mi primerísima tarea profesional.

Recuerdo también sus enseñanzas sobre los detalles. "Fernando" -me decía- "el marco de una puerta debe girar alrededor de todo el vano como lo hace en un cuadro". Hace unos meses, viviendo en un hermoso departamento del s. xix en Uppsala, Suecia y moviéndome por el departamento sin zapatos como se estila en el lugar, se me hacían vivamente presentes esos anchos marcos que sobresalen del nivel del suelo, precisamente porque giran alrededor de todo el vano.

Las palabras de Horacio resonaban entonces frecuentemente en mi memoria, como resonaban también cuando recorría algunas de la obras de Aalto bajo la inconfundible luz de Finlandia. Es que en un lugar tan apartado como Chile, Horacio había acercado a una generación completa de estudiantes a esas realidades arquitectónicas a 
1 Texto leído por Fernando Pérez Oyarzun durante el homenaje celebrado al ex docano Borghes 14 2007 en el campus Lo Contador.

2 La casa estaba en la actual Avda Kennedy, a la altura de Américo

Vespucio. través de su experiencia directa de Mies, de Wright y de Aalto. Hace falta un buen trabajo que describa la importancia que Horacio Borgheresi jugó en el panorama arquitectónico chileno de los años sesenta y comienzos de los setenta. Él es autor de un conjunto de casas -entre ellas la ya mencionada en Lo Saldes y la que proyectó para el doctor Mena en Lo Curro- que se encuentran entre las más refinadas y cualificadas del período. Cuando recién egresado tuve que recomendar a un amigo el mejor arquitecto para encargar una casa no tuve dudas en dar el nombre de Horacio.

No apreció mayormente las aproximaciones intelectuales a la arquitectura. Tenía, en cambio, una aguda sensibilidad y una pasión incontenible por ella. Formó parte de una generación y una cultura para la cual conocimientos y convicciones estaban más cerca de la mano que de la palabra. Fue considerado un representante de lo que Zevi llamaba el pensamiento orgánico, pero tal denominación no termina de definir su actitud frente a la arquitectura. Era un admirador declarado de Wright, lo que no le impedía reconocer sus limitaciones y contradicciones personales. También de Alvar Aalto, cuya obra conocía y contribuyó a difundir. Admiraba la radicalidad de Mies, al que escogió como referencia para el taller que hicimos en el año 1973: sostenía que Mies podía imitarse con provecho, lo que no ocurría con Wright. Partiendo de tales preferencias, sus elaboraciones arquitectónicas tenían sin embargo siempre rasgos de originalidad. A pesar de los cambios que ha experimentado Santiago todavía podemos contemplar los magníficos edificios de vivienda en la costanera Andrés Bello y en la calle Antonio Bellet, como también los que -me parece que en sociedad con Renato Parada- hizo en la esquina de Manuel Montt y Providencia.

Uno de los roles más importantes jugados por Horacio en la universidad, además de sus cargos y su permanente actividad docente, está vinculado a la posibilidad de conectar la formación universitaria con la práctica. No en vano dirigió la Oficina de Prácticas Profesionales donde trabajó con éxito en diversos proyectos significativos junto a algunos de los egresados más talentosos del período. Puede afirmarse que en la base del modelo que escogió para el Departamento de Arquitectura de Obras -enseñar involucrando a los estudiantes en el desarrollo de obras reales- está esa experiencia con las prácticas profesionales. Tal actitud, no pocas veces controvertida, dio origen a una serie de instituciones universitarias que se han ido prolongando bajo distintas formas ya por medio siglo en el ámbito de la facultad. Hoy día, son varias las escuelas en el mundo que han optado por generar organismos que relacionen la academia con la práctica, campo en la que Horacio Borgheresi tuvo una visión premonitoria. Cuando se habla tan insistentemente del diálogo universidad-sociedad y se insiste en las conexiones que debería haber entre investigación y necesidades sociales, no deberían olvidarse esas tempranas intuiciones en un área que no ha logrado como hizo la medicina con el hospital clínico, crear una instancia donde la práctica, la investigación y la enseñanza se combinen.
El alejamiento de Horacio Borgheresi de la Universidad Católica con posterioridad a los acontecimientos políticos y universitarios de 1973, después de un último intento suyo por crear un organismo que vinculara investigación y práctica profesional, fue sin duda difícil para él. Tales circunstancias no le impidieron participar en empresas profesionales, colaborar con el Colegio de Arquitectos de Chile e incluso emprender nuevos proyectos académicos. Su trayectoria arquitectónica y universitaria nos permite afirmar que el tejido cultural de la arquitectura de mediados del s. xx en el Chile, no sería el mismo sin esa hebra representada por la presencia y la figura de Horacio Borgheresi.

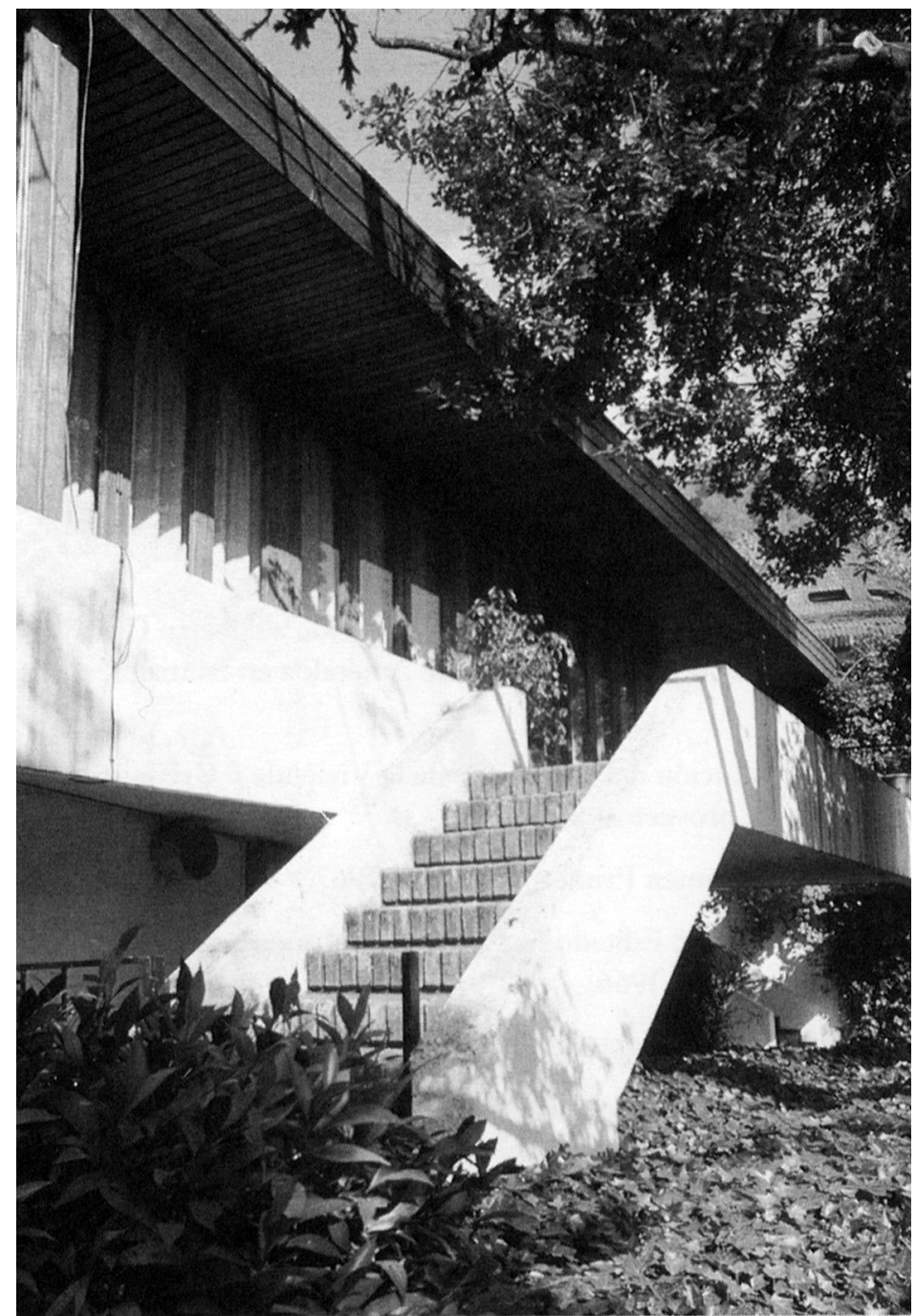

Casa Jordán. Proyecto de Horacio Borgheresi, Providencia, Santiago, 1965. Fotografía de Jaime Ramos. Publicada en Cien años de arquitectura en la Universidad Católica, de Wren Strabucchi, Ediciones ARQ, Santiago, 1994, p. 162 